\title{
Avaliação dos parâmetros físico-químicos de manteigas do tipo comum
}

\author{
Evaluation of the physical-chemical parameters of common butter
}

Regiane Victória de Barros Fernandes ${ }^{[a]}$, Diego Alvarenga Botrel ${ }^{[b]}$, Vivian Raquel de Souza ${ }^{[c]}$,Vitangela Vieira Rocha ${ }^{[c]}$, Celise Souza Ramires ${ }^{[d]}$

[a] Engenheira de alimentos, mestranda em Ciência dos Alimentos pela Universidade Federal de Lavras, técnica de laboratório da Universidade Federal de Viçosa (UFV), Câmpus de Rio Paranaíba, Rio Paranaíba, MG - Brasil, e-mail: regiane.fernandes@ufv.br

[b] Engenheiro de alimentos, mestre em Ciência e Tecnologia de Alimentos pela Universidade Federal de Viçosa (UFV), professor da Universidade Federal de Viçosa (UFV), Câmpus de Rio Paranaíba, Rio Paranaíba, MG - Brasil, e-mail: diego.botrel@ufv.br

[c] Químicas, especialistas, técnicas de laboratório da Universidade Federal de Viçosa (UFV), Câmpus de Rio Paranaíba, Rio Paranaíba, MG - Brasil, e-mail: vivian.souza@ufv.br; vitangela@ufv.br

[d] Acadêmica do curso de Ciências de Alimentos da Universidade Federal de Viçosa (UFV), Câmpus de Rio Paranaíba, Rio Paranaíba, MG - Brasil, e-mail: celise.ramires@ufv.br

\section{Resumo}

As análises físico-químicas de manteiga revelam se a fase oleosa apresenta alterações decorrentes de hidrólise, rancificação ou adulteração e são dados importantes na caracterização da qualidade de manteiga. Para avaliar a qualidade desses produtos, três amostras de oito diferentes marcas de manteigas comercializadas em duas cidades de Minas Gerais (Patos de Minas e Lavras), totalizando 24 amostras, foram submetidas às determinações de umidade, acidez na matéria gorda, substâncias voláteis, sólidos insolúveis e gordura, cinzas, cloretos e reação de Kreis. 0 teor de umidade das amostras variou entre 13,69 e 30,47\%; a acidez, 1,24 e 4,79 mL/100 g de matéria gorda; teor de sólidos insolúveis, 1,95 e 3,65\%; teor de gordura, 67,58 e 84,32\%; cinzas, 0,13 e 4,63\% e cloretos, 0,02 e 0,23\%. Algumas amostras apresentaram resultado positivo para a reação de Kreis, indicando possível rancificação dos produtos. Os resultados indicaram a existência de produtos no mercado sendo comercializados com especificações fora do exigido pela legislação brasileira.

Palavras-chave: Qualidade. Manteiga. Análises físico-químicas. Legislação.

\begin{abstract}
Physico-chemical analyses of butter reveal if its lipidic phase presents changes resultant from hydrolysis, rancidity or adulteration, and therefore are important indicators to characterize the quality of butter. To assess the quality of these products, three samples of eight different brands ( $n=24)$ marketed in two cities of Minas Gerais (Patos de Minas e Lavras) were examined for their moisture content, butter fat acidity, insoluble solids and fat, ash, chlorides and Kreis reaction. The moisture content of samples ranged from 13.69 to 30.47\%, the
\end{abstract}


acidity, 1.24 to $4.79 \mathrm{~mL} / 100 \mathrm{~g}$ of butter fat; insoluble solids content, 1.95 to $3.65 \%$; the fat content ranged from 67.58 to $84.32 \%$; ash, 0.13 to $4.63 \%$ and chlorides at 0.02 to $0.23 \%$. Some samples showed positive result for the Kreis reaction, indicating possible rancidity of the products. These results show that some products have been sold with quality characteristics that do not meet the requirements of the Brazilian Legislation.

Keywords: Quality. Butter. Physico-chemical analysis. Legislation.

\section{Introdução}

A manteiga é tradicionalmente preparada a partir de creme obtido por centrifugação do leite (NONI; RESMINI, 2005) e contém cerca de $15 \%$ de água, $81 \%$ de gordura e geralmente menos de $0,5 \%$ de carboidratos e proteínas (JAY, 2005). Trata-se de um produto gorduroso obtido exclusivamente pela bateção e malaxagem, com ou sem modificação biológica do creme pasteurizado derivado exclusivamente do leite de vaca, por processos tecnologicamente adequados. A matéria gorda da manteiga deverá estar composta exclusivamente de gordura láctea (BRASIL, 1996) e é, dentre os componentes do leite, o principal elemento que entra na fabricação da manteiga (BEHMER, 1999). A manteiga é uma emulsão de água em óleo em que cerca de $16 \%$ de água se encontram na forma de pequenas gotículas esféricas ou ovais incorporadas na fase contínua de gordura (NAHID et al., 2008). No processamento de manteiga, o teor de gordura do leite é concentrado aproximadamente 20 vezes. Em contraste, os outros constituintes do leite, ou seja, proteína, lactose, sais e água são reduzidos (KONAR; HAYALOGLU, 1999).

$\mathrm{Na}$ indústria de alimentos, inocuidade alimentar e qualidade são considerados questões importantes a nível mundial e estão diretamente relacionados com a saúde e o progresso social. Os consumidores procuram cada vez mais produtos alimentícios de marcas confiáveis e esperam que os fabricantes e varejistas forneçam produtos de alta qualidade (GORI et al., 2012).

A qualidade da manteiga depende da qualidade da matéria-prima, tratamento térmico do creme, presença e atividade de microrganismos indesejáveis e procedimentos higiênico-sanitários de processamento e armazenagem (BEHMER, 1999). A deterioração da manteiga pode ser de origem microbiana ou não microbiana. Esta última ocorre graças à oxidação da gordura do leite por mecanismos fotoxidativos ou catalisados por íons metálicos (ARAÚJO, 2004). Os produtos primários da oxidação de ácidos graxos são os hidroperóxidos, compostos altamente reativos que se decompõem rapidamente, originando uma mistura complexa de compostos não voláteis e voláteis tais como hidrocarbonetos, álcoois, ácidos, aldeídos e cetonas, capazes de afetar a qualidade dos produtos (SANCHES-SILVA et al., 2004).

A gordura da manteiga é quase que totalmente constituída de triglicerídeos formados por ácidos graxos saturados de cadeia curta que são susceptíveis à oxidação que altera diversas propriedades como: qualidade, sabor, aroma, textura, cor, valor nutricional e funcionalidade (ARAÚJO, 2004; LOZANO et al., 2007). A oxidação é, portanto, fator importante em relação à vida de prateleira da manteiga (OZKAN et al., 2007). 0 sabor da manteiga é muito apreciado pelo consumidor, o que pode ser confirmado pelo grande número de produtos com aroma de manteiga presentes no mercado (KAYLEGIAN, 1999). Contudo, por causa da presença de grande quantidade de ácidos graxos saturados é extremamente importante manter a qualidade desse produto desde a obtenção da matéria-prima até o uso pelo consumidor.

Assim, o objetivo deste trabalho foi determinar o perfil físico-químico, para verificar a qualidade e a padronização de manteigas comercializadas em duas cidades de Minas Gerais.

\section{Materiais e métodos}

Foram coletadas e analisadas 24 amostras de manteiga tipo "comum com sal" de oito diferentes marcas, sendo quatro marcas comercializadas na cidade de Patos 
de Minas (região do Alto Paranaíba, MG) (marcas A, B, $\mathrm{C}$ e D) e as restantes na cidade de Lavras (região do Sul, MG) (marcas E, F, G e H), escolhidas aleatoriamente e de diferentes lotes e provenientes do comércio local de cada cidade. As amostras foram mantidas refrigeradas até a realização das análises. Todas elas continham o selo do Serviço de Inspeção Federal, estadual ou municipal, estando dentro do prazo de validade.

Foram realizadas análises físico-químicas de matéria gorda em função do teor de umidade e sólidos insolúveis em éter, acidez e cloretos segundo metodologias descritas pela Instrução Normativa n. 68 (BRASIL, 2006) e cinzas e rancidez através da reação de Kreis segundo metodologias descritas pelo Instituto Adolfo Lutz (2005). Todas as análises citadas foram determinadas em triplicata.
Os dados foram submetidos à análise de variância (ANOVA) e Teste de Tukey (nível de 5\% de significância) para avaliar a diferença entre as médias dos dados obtidos. Os resultados foram analisados de acordo com as exigências preconizadas para o produto denominado "manteiga comum" pelo Ministério da Agricultura, Pecuária e do Abastecimento (MAPA) (BRASIL, 2000), de acordo com o Quadro 1.

\section{Resultados}

Os parâmetros físico-químicos umidade, sólidos insolúveis, matéria gorda, acidez, cloretos e cinzas e seus respectivos valores médios e desvios padrão podem ser observados no Quadro 2.

Quadro 1 - Padrões estabelecidos pelo Ministério da Agricultura, Pecuária e do Abastecimento para avaliação da qualidade físico-química de manteigas do tipo comum

\begin{tabular}{lc}
\hline \multicolumn{1}{c}{ Requisitos físico-químicos } & Manteiga comum \\
\hline Teor mínimo de gordura & $80 \%(\mathrm{~m} / \mathrm{m})$ \\
Teor máximo de umidade & $16 \%(\mathrm{~m} / \mathrm{m})$ \\
Teor máximo de cloreto de sódio & $3 \%(\mathrm{~m} / \mathrm{m})$ \\
Acidez máxima & $3 \mathrm{~mL}$ de soluto alcalino normal em $100 \mathrm{~g}$ de gordura láctea no produto \\
Teor máximo de insolúveis no éter etílico & $2 \%^{*}$ \\
\hline
\end{tabular}

Legenda: ${ }^{*}=$ excluído o teor de cloreto de sódio nas variedades salgadas. Fonte: BRASIL (2000).

Quadro 2 - Valor médio e desvio padrão das análises físico-químicas realizadas em amostras de manteiga, comercializadas em Patos de Minas e Lavras (MG)

\begin{tabular}{|c|c|c|c|c|c|c|}
\hline Marcas & $\begin{array}{l}\text { Umidade } \\
(\% \mathrm{~m} / \mathrm{m})\end{array}$ & $\begin{array}{l}\text { Sólidos } \\
\text { insolúveis } \\
(\% \mathrm{~m} / \mathrm{m})\end{array}$ & $\begin{array}{c}\text { Matéria gorda } \\
\qquad \% \mathrm{~m} / \mathrm{m})\end{array}$ & $\begin{array}{c}\text { Acidez na } \\
\text { matéria gorda } \\
(\mathrm{mL} / 100 \mathrm{~g})\end{array}$ & $\begin{array}{l}\text { Cloretos } \\
(\% \mathrm{~m} / \mathrm{m})\end{array}$ & $\begin{array}{l}\text { Cinzas } \\
(\% \mathrm{~m} / \mathrm{m})\end{array}$ \\
\hline$A$ & $19,05 \pm 1,97^{b}$ & $3,65 \pm 0,17^{a}$ & $77,31 \pm 1,97^{b}$ & $3,29 \pm 0,32^{a, b}$ & $0,21 \pm 0,01^{\mathrm{a}}$ & $2,88 \pm 0,90^{a, b}$ \\
\hline B & $15,12 \pm 0,71^{\mathrm{c}}$ & $3,47 \pm 0,05^{a}$ & $81,41 \pm 0,72^{a}$ & $3,58 \pm 0,85^{a}$ & $0,02 \pm 0,01^{\mathrm{e}}$ & $0,20 \pm 0,07^{c}$ \\
\hline$C$ & $14,20 \pm 0,96^{c}$ & $2,55 \pm 0,72^{b}$ & $82,16 \pm 0,94^{a}$ & $3,18 \pm 0,43^{a, b}$ & $0,18 \pm 0,02^{b}$ & $2,12 \pm 0,48^{b}$ \\
\hline D & $15,29 \pm 0,46^{c}$ & $2,04 \pm 0,03^{b, c}$ & $83,76 \pm 0,77^{a}$ & $1,83 \pm 0,99 b$ & $0,22 \pm 0,02^{a}$ & $2,21 \pm 0,08^{b}$ \\
\hline $\mathrm{E}$ & $20,26 \pm 0,26^{b}$ & $2,43 \pm 0,50^{b, c}$ & $77,31 \pm 0,54^{b}$ & $4,10 \pm 0,15^{a}$ & $0,13 \pm 0,01^{\mathrm{c}, \mathrm{d}}$ & $3,46 \pm 1,66^{a}$ \\
\hline $\mathrm{F}$ & $27,51 \pm 2,71^{\mathrm{a}}$ & $1,95 \pm 0,03^{c}$ & $70,54 \pm 2,71^{c}$ & $4,25 \pm 0,29^{a}$ & $0,15 \pm 0,02^{c}$ & $0,94 \pm 0,71^{\mathrm{c}}$ \\
\hline G & $25,60 \pm 1,26^{a}$ & $2,55 \pm 0,30^{b}$ & $71,84 \pm 1,00^{c}$ & $3,09 \pm 0,08^{a, b}$ & $0,14 \pm 0,02^{c, d}$ & $2,53 \pm 0,16^{a, b}$ \\
\hline $\mathrm{H}$ & $14,06 \pm 0,26^{c}$ & $2,16 \pm 0,12^{b, c}$ & $83,78 \pm 0,27^{a}$ & $1,97 \pm 0,49 b$ & $0,12 \pm 0,01^{\mathrm{d}}$ & $1,13 \pm 0,46^{b, c}$ \\
\hline
\end{tabular}

Fonte: Dados da pesquisa.

Nota: ${ }^{a-e}=$ médias seguidas por letras diferentes na mesma coluna diferem entre $\mathrm{si}(\mathrm{p}<0,05)$. 
Os resultados do teste químico de Kreis são verificados no Quadro 3.

Quadro 3 - Resultados do teste químico qualitativo de Kreis em manteiga comercializada em Patos de Minas e Lavras (MG)

\begin{tabular}{cccc}
\hline & \multicolumn{3}{c}{ Resultados } \\
\cline { 2 - 4 } Amostras & Lote 1 & Lote 2 & Lote 3 \\
\hline A & - & - & - \\
B & + & + & + \\
C & - & + & + \\
D & + & - & + \\
E & - & - & - \\
F & - & - & - \\
G & - & - & - \\
H & - & - & - \\
\hline
\end{tabular}

Fonte: Dados da pesquisa.

\section{Discussão}

As amostras de diferentes marcas de manteiga analisadas apresentaram significativa variação nos parâmetros estudados, encontrando-se algumas delas fora dos padrões exigidos pela legislação. A umidade das manteigas variou de 13,69 a 30,47\% (m/m) estando acima do exigido pela legislação (máximo de $16 \%$ ) nas manteigas das marcas A, E, F e G. As marcas do sul de Minas Gerais tiveram uma maior representação negativa em relação a este parâmetro. 0 excesso de água na manteiga constitui fraude, pois o consumidor está sendo lesado ao adquirir mais água do que é exigido pela legislação. Além disso, é um indicativo da falta de controle nas etapas de batedura e malaxagem do creme (BEHMER, 1999). Os valores de umidade verificados por Augusta e Santana 1998, em estudo envolvendo manteigas comercializadas na cidade do Rio de Janeiro (RJ), variaram de 11,31 a $19,97 \%$ e de 11,51 a $23,97 \%$ em diferentes marcas (Silva et al. (2009), estando 50,00\% das amostras em desacordo com o padrão (AUGUSTA; SANTANA, 1998), valor próximo ao observado por Silva et al. (2009) (46,66\%).

Em relação aos sólidos insolúveis em éter, em que o valor máximo exigido é de $2 \%$, todas as amostras das marcas A, B e G, duas da marca C e uma das marcas $\mathrm{E}$ e $\mathrm{H}$ estavam em desacordo com o padrão vigente, estando $54 \%$ das amostras avaliadas, fora do valor determinado por lei. Silva et al. (2009) também encontraram grandes variações nesse parâmetro, observando valores entre 0,56 e 6,25\%, em manteigas comercializadas na cidade de Viçosa (MG).

Relativamente ao teor de matéria gorda, o percentual desse parâmetro variou entre 67,58 a $84,32 \%(\mathrm{~m} / \mathrm{m})$. Valores similares foram verificados por Silva et al. (2009) $(68,36$ a $85,85 \%)$ na caracterização de manteigas comercializadas na cidade de Viçosa (MG). As três amostras analisadas da marca A, E, F e G estavam com o parâmetro abaixo dos $80 \%$ exigidos por lei, perfazendo $50 \%$ de todas as amostras analisadas. No estudo de Silva et al. (2009), $37 \%$ das amostras analisadas de manteiga estavam também em desacordo com o padrão vigente. Os autores concluíram que a variação na concentração de matéria gorda pode ter ocorrido por causa da falta de padronização de procedimentos operacionais, ou ainda em razão da falta de controles da composição durante o processamento.

Com relação à acidez nos produtos, foram encontrados valores acima de $3 \mathrm{~mL} / 100 \mathrm{~g}$ de matéria gorda em todas as amostras das marcas $\mathrm{E}$ e $\mathrm{F}$, em duas amostras das as marcas A, B e G e em uma amostra da marca C, estando as amostras das marcas D e $\mathrm{H}$ com acidez dentro do valor recomendado. A presença de alta acidez é um indicativo de deterioração da manteiga e que pode gerar rejeição do produto dependendo do estágio em que se apresenta. Apesar de a acidez poder variar naturalmente com a vida de prateleira da manteiga, as condições exigidas devem estar dentro da faixa especificada pelos órgãos competentes durante a validade do produto. Nesse estudo, $54 \%$ das amostras estavam fora do padrão exigido. Para esse mesmo quesito, Silva et al. (2009) encontraram $13,3 \%$ das amostras com valores acima do padrão. Já Maziero et al. (2010) e Augusta e Santana (1998) observaram que todas as amostras analisadas estavam dentro dos limites estabelecidos. Esse índice pode indicar a atividade proteolítica de bactérias produtoras de lipases, enzimas indesejáveis ao creme, responsáveis por modificações sensoriais e que podem reduzir a qualidade do produto e a vida de prateleira (OLIEMAN; VAN RIEL, 1989; SILVA et al., 2009; VAN RIEL; OLIEMAN, 1995).

Os teores de cloreto expressos em \% de cloreto de sódio/100 g de amostra, estiveram abaixo do 
limite máximo estabelecido (3\%) em todas as amostras. Semelhantes resultados foram encontrados por Silva et al. (2009) nas manteigas analisadas do tipo comum. Porém, para as marcas do tipo extra, estudadas por Augusta e Santana (1998) e por Silva et al. (2009), respectivamente $7,5 \%$ e $2,88 \%$, apresentaram teor de $\mathrm{NaCl}$ acima dos padrões, em desacordo com a regulamentação que é de no máximo $2 \%$ para esse tipo de manteiga.

Os teores de cinzas (valores limites não especificados na legislação) igualmente apresentaram grande variação entre as amostras e, acompanhado dos outros parâmetros estudados, constituíram indicativo de que manteigas podem se diferenciar muito entre uma marca e outra, inclusive dentro de uma mesma marca. No entanto, deve-se reforçar a importância da manutenção dos valores das variáveis de interesse dentro dos limites estabelecidos pela legislação.

Maziero et al. (2010) observaram resultado negativo para rancidez por meio da reação de Kreis, dentre as amostras demanteigaanalisadas. No presente estudo, foi encontrado resultado positivo nas amostras da marca B e para duas amostras das marcas C e D. 0 resultado positivo do método de Kreis é indicativo de rancidez no produto, oriunda da ação do oxigênio do ar ou de microrganismos, provocando alterações no odor e no sabor de manteigas, depreciando o produto.

\section{Conclusões}

De acordo com os parâmetros avaliados neste estudo (exceção da marca D, comercializada na cidade de Patos de Minas, MG), todas as manteigas analisadas neste trabalho encontravam-se com pelo menos um parâmetro de qualidade fora dos padrões definidos pela legislação brasileira, o que mostra a necessidade de padronização do processo de fabricação deste produto pelas indústrias. Tal fato evitará que o consumidor seja lesado, além de garantir a comercialização de produtos com maior qualidade.

\section{Referências}

ARAÚJO, J. M. A. Química de alimentos teoria e prática. 3 ed., Viçosa: Editora UFV, 2004.
AUGUSTA, I. M.; SANTANA, D. M. N. Avaliação da qualidade de manteigas tipo extra comercializadas no Estado do Rio de Janeiro. Ciência e Tecnologia de Alimentos, v. 18, n. 4, 1998.

BEHMER, M. L. A. Tecnologia do leite: queijo, manteiga, caseína, iogurte, sorvetes e instalações: produção, industrialização, análise. 13 ed. São Paulo: Nobel, 1999.

BRASIL. Instrução Normativa n. 68 de 12 de dezembro de 2006 do Ministério da Agricultura, Pecuária e Abastecimento. Diário Oficial (da) União, Poder Executivo, Brasília, DF, 14 de dezembro de 2006. Seção 1, p. 8. Secretaria de Defesa Agropecuária. Departamento de Inspeção de Produtos de origem Animal. Métodos Analíticos Oficiais Físico-Químicos para Controle de Leite e Produtos Lácteos.

BRASIL. Resolução n. 4 de 28 de junho de 2000 do Ministério da Agricultura Pecuária e Abastecimento. Diário Oficial (da) União, Poder Executivo, Brasília, DF, de 5 de julho de 2000. Seção 1, p. 5. Resolução para denominação de manteiga comum comercializada em território nacional.

BRASIL. Portaria n. 146 de 7 de março de 1996 do Ministério da Agricultura Pecuária e Abastecimento. Diário Oficial (da) União, Poder Executivo, Brasília, DF, de 11 de março de 1996. Seção 1, p. 3977. Regulamento Técnico de Identidade e Qualidade da Manteiga.

GORI, A. et al. A rapid method to discriminate season of production and feeding regimen of butters based on infrared spectroscopy and artificial neural networks. Journal of Food Engineering, v. 109, p. 525-530, 2012.

INSTITUTO ADOLFO LUTZ. Métodos físico-químicos para análise de alimentos. 4. ed. Brasília: Ministério da Saúde, 2005.

JAY, J. M. Microbiologia de Alimentos. 6. ed. Porto Alegre: Artmed, 2005.

KAYLEGIAN, K. E. The production of specialty milk fat ingredients. Journal Dairy Science, v. 82, n. 7, p. 1433 1439, 1999.

KONAR, A.; HAYALOGLU, A. Tereyag Uretim Yontemleri, KremaveYogurttan Eldeedilen Tereyaglarin Fizikokimyasal, Mikrobiyolojik ve duyusal Nitelikleri-II. Standard, v. 448, p. 53-58, 1999.

LOZANO, P. R et al. Effect of cold storage and packaging material on the major aroma components of sweet cream butter. Journal of Agricultural and Food Chemistry, v. 55, p. 7840-7846, 2007. 
MAZIERO, M. T. et al. Microrganismos psicrotróficos lipolíticos em produtos lácteos durante o prazo comercial. Revista do Instituto de Laticínios Cândido Tostes, n. 372 , v. 65 , p. $30-35,2010$.

NAHID, A. et al. Modelling the freezing of butter. International Journal of Refrigeration, v. 31, p. 152-160, 2008.

NONI, I.; RESMINI, P. Identification of rennet-whey solids in "traditional butter" by means of HPLC/ESI-MS of non-glycosylated caseinomacropeptide A. Food Chemistry, v. 93, p. 65-72, 2005.

OLIEMAN, C.; VAN RIEL, J. A. M. Detection of rennet whey solids in skim milk powder and buttermilk with reverse-phase HPLC. Netherlands Milk and Dairy Journal, v. 43, p. 171-184, 1989.

OZKAN, G. et al. Antioxidant activities of Satureja cilicica essential oil in butter and in vitro. Journal of Food Engineering, v. 79, p. 1391-1396, 2007.
SANCHES-SILVA, A. S. et al. Determination of hexanal as indicator of lipid oxidation state in potato crisps using gas chromatography and high-performance liquid chromatography. Journal of Chromatography, v. 1046, p. 7581, 2004.

SILVA, L. C. A. et al. Estudo da qualidade de manteigas por fosfatase alcalina e análises físico-químicas. Revista do Instituto de Laticínios Cândido Tostes, v. 64 , n. 367-368, p. 42-47, 2009.

VAN RIEL, J.; OLIEMAN, C. Determination of caseinomacropeptide with capillary zone electrophoresis and its application to the detection and estimation of rennet whey solids in milk and buttermilk powder. Electrophoresis, v. 16, p. 529-533, 1995.

Recebido: $17 / 07 / 2012$

Received: 07/17/2012

Aprovado: 23/10/2012

Approved: 10/23/2012 